\title{
Schlachtwert und Fleischqualität von Lämmern der Schafrasse Skudden
}

\author{
HENRYK BRZOSTOWSKI, STANISŁAW MILEWSKI und ZENON TAŃSKI
}

Wydział Bioinżynierii Zwierząt, Uniwersytet Warmińsko-Mazurski Olsztyn, Polen

\section{Zusammenfassung}

Die Untersuchungen wurden mit 12 Lämmern der Skudden-Rasse im Alter von 100 Tagen durchgeführt. Das Untersuchungsziel lag in der Bestimmung des Schlachtwertes und der Fleischqualität dieser Tiere. Es wurden folgende Merkmale erfasst: Lebendmasse, Schlachtleistung, "Lendenmuskelfläche", Nierenfettmenge, Anteile von Keule, Rücken, Entrecote am Schlachtkörper sowie die Gewebezusammensetzung der Keulen. Die Fleischqualität wurde anhand entnommener Proben aus dem Oberschenkelmuskel (M. quadriceps femoris) bewertet. In diesen Proben wurden bestimmt: Trockenmassegehalt, Rohproteingehalt, Rohfett, Rohaschegehalt, pH-Wert nach 24 h, Wasser-Eiweiß-Index, Bruttoenergiegehalt (Brennwert), Wasseraufnahmefähigkeit, Farbhelligkeit, Muskelfaserdurchmesser, Cholesteringehalt, Vitamin A- und E-Gehalt, Aminosäurenprofil, Fettsäurenmuster des intramuskulären Fettes sowie sensorische Merkmale.

Anhand der durchgeführten Untersuchungen konnte feststellt werden, dass der Schlachtwert der Skudden-Lämmer ein relativ gutes Niveau aufweist. Die Lebendmasse vor der Schlachtung $(12,56 \mathrm{~kg})$ ist zwar niedrig, aber der Schlachtkörper zeichnet sich durch eine geringe Verfettung sowie eine günstige Gewebezusammensetzung der Keulen (76,38\% Fleisch, 5,68\% Fett und 17,94\% Knochen) aus. Der Schlachtleistungsindex beträgt 44,93\% und der Anteil wertvoller Teilstücke an den Schlachthälften 38,77\%. Das Fleisch von 100 Tage alten Skudden-Lämmern ist von hoher Qualität. Es weist eine dunkle Farbe und spezifische sensorische Merkmale auf, die mit Wildbret vergleichbar sind. Ein niedriger Fett- $(1,56 \%)$ und Cholesteringehalt $(34,47 \mathrm{mg} / 100 \mathrm{~g})$, eine hohe Vitamin A-Konzentration $(5,25 \mu \mathrm{g} / \mathrm{g})$ sowie ein günstiges Fettsäurenprofil im intramuskulären Fett zeugen von wertvollen gesundheitlichen Merkmalen dieses Fleisches. Die feinfaserige Muskelstruktur mit einem Faserdurchmesser von 17,24 $\mu \mathrm{m}$ lässt schlussfolgern, dass dieses Fleisch leicht verdaulich und gut bekömmlich ist. Aufgrund der erzielten Ergebnisse wird empfohlen, diese Rasse wegen ihrer besonderen Fleischmerkmale in größerem Umfang für die Mastlämmerproduktion zu halten.

Schlüsselwörter: Schafe, Skudden, Mastlämmer, Schlachtwert, Fleischqualität

\section{Abstract}

\section{Slaughter value and meat quality of Skudde lambs}

The investigations were conducted on 100-day-old lambs of the Skudde breed and were aimed at determining the slaughter value of lambs and quality of their meat. The slaughter value was evaluated based on: body weight before slaughter, dressing percentage, loin 
"eye« area, perinephric fat content, contribution of valuable cuts in the carcass (leg, loin, steak) and tissue composition of legs. In turn, meat quality was assessed based on samples collected from the muscle of the thigh (M. quadriceps femoris) and included: dry matter, total protein, crude fat, crude ash, $\mathrm{pH}$ after $24 \mathrm{~h}$, water/protein index, energy value, water holding capacity, colour brightness, muscle fibre diameter, levels of cholesterol, vitamin A and E, protein amino acid profile, fatty acid composition of intramuscular fat, as well as sensory attributes, namely: aroma, taste, juiciness and tenderness. Analyses conducted in the study demonstrated that the slaughter value of the lambs was at a relatively good level. A low body weight before slaughter ( $12.56 \mathrm{~kg}$ ) was compensated for by very low adiposity of carcasses and by a favourable tissue composition of legs, i.e. $76.38 \%$ of meat, $5.68 \%$ of fat and $17.94 \%$ of bones. Dressing percentage accounted for $44.03 \%$, and the percentage of primal cuts in the half-carcass was $38.77 \%$. Meat of the 100-day-old Skudde lambs was found to be of high quality. It was characterized by dark colour and specific sensory attributes, which makes it comparable to game meat. Low contents of fat $-1.56 \%$ and cholesterol $-34.47 \mathrm{mg} / 100 \mathrm{~g}$, a high concentration of vitamin $\mathrm{A}-5.25 \mu \mathrm{g} / \mathrm{g}$ as well as a favourable fatty acid profile of intramuscular fat indicate high health-promoting values of that meat. In addition, the finefibrous structure of muscles (fibre diameter of $17.24 \mu \mathrm{m}$ ) suggests that the meat is light and easy digestible. The results obtained in the study substantiate the advisability of a wider application of this breed for meat production purposes.

Keywords: sheep, Skudde, fattening lambs, slaughter value, meat quality

\section{Einleitung}

Die Schafrasse "Skudden« war schon im Mittelalter in Ostpreußen und in den Gebieten des Baltikums bekannt. Nach ŚLIWA (1957) weist diese Schafrasse ähnliche Merkmale wie die Graue Heidschnucke auf. Skudden werden der Gruppe kurzschwänziger polnischer und litauischer Heideschafrassen, deren Abstammung vom europäischen Muflon abgeleitet wird, zugeordnet. Die Skudden-Schafe aus Ostpreußen werden für die älteste und kleinste nordische Haustierschafrasse (NUERNBERG et al. 2008) gehalten. Im Jahre 1873 belief sich der Skuddenbestand in Ostpreußen auf ca. 77000 Stück. Die schnelle landwirtschaftliche Entwicklung nach dem ersten Weltkrieg führte zu einer rapiden Bestandreduzierung dieser kleinen Landschafrasse einerseits und einer Bestandzunahme sowohl beim (edlen) Schwarzköpfigen Fleischschaf als auch beim Württemberger Schaf andererseits. Im Jahre 1936 betrug der Skudden-Bestand auf dem Gebiet von Ostpreußen nur noch 3621 reinrassige Tiere (SAMBRAUS 1996). Auf Grund der Folgen des 2. Weltkrieges war diese Schafrasse fast ausgestorben. Nur eine geringe Tierzahl überlebte und dies dank passionierter Schafzüchter sowie den Bemühungen von Tiergärten (u.a. in Leipzig, KRISCHE 1992). In Deutschland wurde diese Rasse auf die Rote Liste gefährdeter Arten der nordischen Schafe gesetzt (DUCHEV et al. 2006). Der 1984 in Frankfurt am Main gegründete Zuchtverband der Skuddenschafe wurde beauftragt, diese Rasse als eine sehr wertvolle Genreserve zu erhalten. In den zur Verfügung stehenden Publikationen (KNABE et al. 1988, FISCHER et al. 1994, SAMBRAUS 1996, KURT und BRANDT 2008) sind ausführliche Informationen zum Skuddenexterieur, den Standortanforderungen, der Lebendmasse und Körpermaße, den quantitativen und qualitativen Wollmerkmalen sowie der Zuchtleistung enthalten. Wenige Daten wurden 
jedoch bisher zum Schlachtwert und zur Fleischqualität der Lämmer dieser Schafrasse veröffentlicht. Deshalb wurden entsprechende Untersuchungen durchgeführt, über deren Ergebnisse nachfolgend berichtet wird.

\section{Material und Methoden}

Die Untersuchungen wurden an 12 männlichen Einlingslämmern der Schafrasse Skudden aus der Herde Oczkowski in Wężówka, Polen durchgeführt. Die Herde stammt aus der Biologischen Station Lippe der Universität Bielefeld, Deutschland. Die Übergabe der Tiere erfolgte $2004 \mathrm{im}$ Rahmen des Projekts "SKUDDEN« der Europäischen Union zwecks Prüfung, wie sich diese Schafe in den Gebieten verhalten, die ehemals ihre natürlichen Standorte waren sowie zwecks Erweiterung ihres Bestandes. Die in die Untersuchungen einbezogenen Lämmer stammten aus der Ablammung im Januar 2008. Neben der Muttermilch erhielten die Lämmer ab dem 14. Tag als weitere Futtermittel Wiesenheu und geringe Hafermengen. Im Alter von 100 Tagen wurden die Tiere geschlachtet. Bei der Bewertung des Schlachtwertes wurden die folgenden Parameter berücksichtigt: Lebendmasse vor der Schlachtung, Schlachtleistung, "Lendenmuskelfläche«, Nierenfettmenge, Anteil wertvoller Teilstücke am Schlachtkörper (Keule, Rücken, Hochrippe) sowie die Gewebezusammensetzung der Keulen (NAWARA et al. 1963). Zur Bewertung der Fleischqualität wurden Proben aus dem Oberschenkelmuskel (M. quadriceps femoris) entnommen und den folgenden Analysen unterzogen: Trockenmasse durch Trocknung der Proben bei einer Temperatur von $105^{\circ} \mathrm{C}$; Rohprotein nach der KjeldahlMethode; Rohfett nach der Soxhlet-Methode; Rohasche durch Verbrennung bei einer Temperatur von $550^{\circ} \mathrm{C}$; Brennwert (Bruttoenergie) mit Hilfe des adiabatischen Kaloriemeters KL-10 mit eingebauter Bombe; Durchmesser der Muskelfasern, indem das Mikroskopbild der Präparate, die nach der in der Arbeit von BRZOSTOWSKI und TAŃSKI (2006) beschriebenen Methode vorbereitet wurden, einer Computeranalyse unterzogen wurden; Wasserbindung nach der Methode von GRAU und HAMM (1953); Farbhelligkeit mit dem Spektrokolorimeter "Specol« mit Zusatzgerät R 045 bei einer Wellenlänge von 560 nm; pH-Wert 24 Stunden nach der Schlachtung mit dem pH-Meter der Firma Radiometer PHM 22; Cholesteringehalt mit dem Kolorimeter EPOLL 20 nach der durch RHEE et al. (1982) beschriebenen Methode; Vitamin A- und Vitamin E-Gehalt mit der HPCL-Methode auf dem Chromatographen Shimadzu (RETTENMAIER und SCHÜEP 1992); Aminosäurenprofil des Eiweißes mit dem automatischen Analysator für Aminosäuren AAA-T-339M, Hersteller Mikrotechna (die S-haltigen AS Cystin und Methionin wurden als Cysteinsäure und Methioninsulfon bestimmt); Fettsäurenmuster des intramuskulären Fetts nach der Esterifizierungsmethode (PEISKER 1964) und anschließend gaschromatographisch unter Verwendung des Chromatographen PYE Unicam, Serie 104 mit dem Flammenionisationsdedektor als Glassäule mit einer Länge von 2,1 m und einem Innendurchmesser von $4 \mathrm{~mm}$ (ŻEGARSKA et al. 1979); sensorische Fleischmerkmale wie Geruch (Intensität und Erwartungshaltung), Schmackhaftigkeit (Intensität und Erwartungshaltung), Saftigkeit und Zartheit nach thermischer Verarbeitung laut Vorschrift von BARYŁKO-PIKIELNA (1975). Die sensorische Bewertung führten fünf Personen unter Anwendung einer Punkteskala (1 bis 5 Punkte) durch.

Die Mittelwerte und Standardabweichungen wurden statistisch mittels Statistika 8.0 for Windows berechnet (StatSoft Inc. 2007). 


\section{Ergebnisse}

Nach 100 Lebenstagen hatten die untersuchten Lämmer eine mittlere Lebendmasse von 12,56 $\pm 0,51 \mathrm{~kg}$ erreicht und die Schlachtleistung lag im Mittel bei 44\% (Tabelle 1). Die Schlachtkörper zeichneten sich durch eine geringe Verfettung aus, was sowohl durch den niedrigen Nierenfettanteil an der Schlachtkörpermasse $(0,82 \%)$ als auch durch die Gewebezusammensetzung der Keulen eindeutig dokumentiert wird. Der Anteil wertvoller Teilstücke an der Schlachtkörpermasse belief sich auf 38,77\%. Das wertvollste Teilstück, die Keule, hatte einen niedrigen Fettanteil (5,68\%) bei gleichzeitig hohem Fleischanteil (76\%). Daraus ergibt sich ein sehr weites Fleisch/ Fett-Verhältnis von 14:1.

Tabelle 1

Lebendmasse und Schlachtwert

Body weight and slaughter value

\begin{tabular}{lccc}
\hline Parameter & & Mittelwert & SD \\
\hline Lebendmasse vor der Schlachtung & $\mathrm{kg}$ & 12,56 & 0,51 \\
Schlachtkörpermasse & $\mathrm{kg}$ & 5,53 & 0,25 \\
Schlachtleistung & $\%$ & 44,03 & 0,41 \\
Nierenfettanteil & $\%$ & 0,82 & 0,15 \\
Rechte Schlachthälftemasse & $\mathrm{kg}$ & 2,76 & 0,12 \\
Wertvolle Teilstücke an der Schlachthälfte & $\mathrm{kg}$ & 1,07 & 0,04 \\
& $\%$ & 38,77 & 1,57 \\
"Lendenmuskelfläche« & $\mathrm{cm}$ & 8,28 & 1,62 \\
Keule: & $\mathrm{kg}$ & 0,73 & 0,03 \\
- Fleisch & $\mathrm{kg}$ & 0,56 & 0,03 \\
& $\%$ & 76,38 & 0,49 \\
- Fett & $\mathrm{kg}$ & 0,04 & 0,01 \\
& $\%$ & 5,68 & 0,58 \\
- Knochen & $\mathrm{kg}$ & 0,13 & 0,01 \\
& $\%$ & 17,94 & 0,51 \\
Fleisch/Knochen - Verhältnis & & 4,31 & 0,14 \\
Fleisch/Fett - Verhältnis & & 14,00 & 1,48 \\
\hline
\end{tabular}

Die analytisch ermittelten Qualitätseigenschaften des Lammfleisches sind in Tabelle 2 zusammengestellt. Das Fleisch ist relativ proteinreich (19,28\% Rohprotein) und enthält wenig Fett (1,56\%). Es zeichnet sich durch eine dunkle Farbe und einen geringen Muskelfaserdurchmesser $(7,24 \mu \mathrm{m})$ sowie eine niedrige Cholesterinkonzentration $(34,47 \mathrm{mg} /$ $100 \mathrm{~g}$ ) aus. Der Vitamine A- bzw. Vitamin E-Gehalt in $1 \mathrm{~g}$ Muskelgewebe beträgt 5,25 bzw. $0,34 \mu \mathrm{g}$.

Beim Aminosäureprofil des Fleischproteins (Tabelle 3) überwiegen leicht die nichtessenziellen Aminosäuren (53,32\%), unter innen die Glutaminsäure mit $14,86 \%$, die Asparaginsäure mit 9,45\% sowie Arginin mit 6,99\% im Rohprotein. Von den essenziellen Aminosäuren sind Lysin mit 8,59\% und Leuzin mit 8,52\% am höchsten im Fleischprotein enthalten. Das Verhältnis lebensnotwendige zu entbehrliche Aminosäuren beträgt 0,8:1,0.

Im intramuskulären Fett wurden 16 Fettsäuren mit einer Kettenlänge von $C_{12}$ bis $C_{20}$ analysiert (Tabelle 4). Auf die gesättigten Säuren entfallen 49,81\%. In dieser Gruppe überwiegen folgende Fettsäuren: $C_{16: 0}$ mit $25,08 \%$ und $C_{18: 0}$ mit $15,15 \%$. In der Gruppe der 
ungesättigten Fettsäuren dominieren die einfach ungesättigten Säuren mit 42,63\%, unter innen $C_{18: 1}$ mit 37,64\%. Von den analysierten Fettsäuren entfallen 65,34 \% auf die Gruppe der DFA (UFA $\left.+C_{18: 0}\right)$. Dadurch ergibt sich ein günstiges DFA/OFA-Verhältnis von 1,9:1.

Tabelle 2

Qualitätseigenschaften des Lammfleisches

Quality traits of meat

\begin{tabular}{lccc}
\hline Parameter & & Mittelwert & SD \\
\hline Trockenmasse & $\%$ OS* & 22,20 & 0,16 \\
Rohprotein & $\%$ OS & 19,28 & 0,13 \\
Rohfett & $\%$ OS & 1,56 & 0,16 \\
Rohasche & $\%$ OS & 1,22 & 0,05 \\
$\mathrm{pH}$ - Wert nach 24 h & 5,86 & 0,05 \\
Wasser/Eiweiß - Verhältnis & 4,08 & 0,06 \\
Energiewert (Brennwert) & $\mathrm{kcal} / 100 \mathrm{~g}$ Fleisch & 121,49 & 1,11 \\
Farbenhelligkeit & $\%$ & 13,75 & 0,38 \\
Wasseraufnahmefähigkeit & $\mathrm{cm}^{2}$ & 7,00 & 0,31 \\
Muskelfaserdurchmesser & $\mu \mathrm{m}$ & 17,24 & 1,07 \\
Cholesterin & $\mathrm{mg} / 100 \mathrm{~g}$ Fleisch & 34,47 & 1,68 \\
Vitamin A & $\mu \mathrm{g} / \mathrm{g}$ Fleisch & 5,25 & 0,46 \\
Vitamin E & $\mu \mathrm{g} / \mathrm{g}$ Fleisch & 0,34 & 0,07 \\
\hline
\end{tabular}

"OS Originalsubstanz

Tabelle 3

Aminosäurenmuster des Fleischproteins, \% (im Rohprotein)

Amino acid profile of protein in meat, \% (of the crude protein)

\begin{tabular}{lcc}
\hline Aminosäuren & Mittelwert & SD \\
\hline Threonin & 4,49 & 0,01 \\
Valin & 5,25 & 0,08 \\
Methionin & 2,47 & 0,01 \\
Isoleucin & 4,93 & 0,02 \\
Leucin & 8,52 & 0,03 \\
Phenylalanin & 3,77 & 0,01 \\
Histidin & 2,72 & 0,01 \\
Lysin & 8,59 & 0,01 \\
Tryptophan & 1,35 & 0,01 \\
Summe der essenziellen Aminosäuren & 42,09 & 0,02 \\
Asparaginsäure & 9,45 & 0,03 \\
Serin & 3,84 & 0,01 \\
Glutaminsäure & 14,86 & 0,05 \\
Prolin & 3,79 & 0,01 \\
Cystin & 1,19 & 0,01 \\
Glyzin & 4,35 & 0,01 \\
Alanin & 5,29 & 0,16 \\
Tyrosin & 3,56 & 0,01 \\
Arginin & 6,99 & 0,02 \\
Summe der nichtessenziellen Aminosäuren & 53,32 & 0,03 \\
Verhältnis essenzielle/nichtessenzielle Aminosäuren & 0,80 & 0,02 \\
\hline
\end{tabular}


Tabelle 4

Fettsäurenprofil des intramuskulären Fetts (in \% der Gesamtfettsäuren)

Fatty acid composition in intramuscular fat (\% of the total fatty acids)

\begin{tabular}{lcc}
\hline Fettsäuren & Mittelwert & SD \\
\hline$C_{12: 0}$ & 0,91 & 0,04 \\
$C_{14: 0}$ & 6,39 & 0,32 \\
$C_{15: 0}$ & 0,84 & 0,02 \\
$C_{16: 0}$ & 25,08 & 0,55 \\
$C_{17: 0}$ & 1,26 & 0,02 \\
$C_{18: 0}$ & 15,15 & 0,60 \\
$C_{20: 0}$ & 0,18 & 0,03 \\
Summe der gesättigten Fettsäuren (SFA) $_{C_{14: 1}}$ & 49,81 & 0,24 \\
$C_{16: 1}$ & 0,43 & 0,05 \\
$C_{17: 1}$ & 3,15 & 0,09 \\
$C_{18: 1}$ & 0,83 & 0,04 \\
$C_{20: 1}$ & 37,64 & 0,52 \\
Summe der einfach ungesättigten Fettsäuren (MUFA) & 0,58 & 0,04 \\
$C_{18: 2}$ & 42,63 & 0,26 \\
$C_{18: 3}$ & 5,41 & 0,29 \\
$C_{20: 2}$ & 0,79 & 0,06 \\
$C_{20: 4}$ & 0,22 & 0,03 \\
Summe der mehrfach ungesättigten Fettsäuren (PUFA) & 1,14 & 0,06 \\
Summe der ungesättigten Fettsäuren (UFA) & 7,56 & 0,19 \\
UFA/SFA & 50,19 & 0,21 \\
PUFA/MUFA & 1,01 & 0,02 \\
PUFA/SFA & 0,18 & 0,01 \\
DFA (UFA+C & 0,15 & (18:0 \\
OFA (SFA-C & 65,34 & 0,01 \\
DFA/OFA & 34,66 & 0,88 \\
\hline & 1,89 & 0,88 \\
& & 0,07 \\
\hline
\end{tabular}

Tabelle 5

Sensorische Bewertung des Lammfleisches (Noten)

Sensory evaluation of meat (points)

\begin{tabular}{lcc}
\hline Parameter & Mittelwert & SD \\
\hline Geruch - Intensität & 4,83 & 0,07 \\
Geruch - Erwartungshaltung & 4,63 & 0,12 \\
Geschmack - Intensität & 4,67 & 0,11 \\
Geschmack - Erwartungshaltung & 4,75 & 0,11 \\
Saftigkeit & 4,46 & 0,18 \\
Zartheit & 4,38 & 0,20 \\
Durchschnittsnote & 4,62 & 0,13 \\
\hline
\end{tabular}

Die Ergebnisse der sensorischen Fleischbewertung enthält Tabelle 5. Die Durchschnittsnote für die bewerteten Merkmale beträgt 4,62 Punkte. Die Merkmale Geschmack und Geruch wurden etwas höher als die anderen Parameter bewertet. Die höchsten Noten wurden für die Geruchintensität und Geschmackerwartungshaltung vergeben (4,83 bzw. 4,75 Punkte) und die niedrigsten für Zartheit und Saftigkeit (4,38 bzw. 4,46 Punkte). 


\section{Diskussion}

Bei der Bewertung des Schlachtwertes von 100 Tage alten Lämmern der Schafrasse Skudden ist darauf hinzuweisen, dass ihre niedrige Lebendmasse als Nachteil einzustufen ist. Sie liegt unter $50 \%$ der Lebendmasse gleichaltriger Lämmer anderer Rassen, wie z. B. des polnischen (Kamieniecka) Langwollschafes (BRZOSTOWSKI et al. 1999, SZCZEPAŃSKI et al. 2002b), die unter ähnlichen Bedingungen in der Region Ermland und Masuren gehalten werden. Vergleichbare Lebendmassen werden durch die Lämmer weiterer Landschafrassen wie z. B. vom Polnischen Bergschaf, Weißem Alpenschaf (PARAPONIAK und ROBORZYŃSKI 2001, PARAPONIAK und KAWECKA 2004, POMPA-ROBORZYŃSKI und KĘDZIOR 2006) und Grauer Gehörnter Heidschnucke (SEIBERT et al. 2004) erreicht. Wie bei anderen Landschafrassen, z. B. bei der Kamienieckarasse (SZCZEPAŃSKI et al. 2002b), beträgt in der vorliegenden Untersuchung die Schlachtausbeute bei den Skudden-Lämmern 44\% bei gleichzeitig guter Bemuskelung. Charakteristisch ist die sehr niedrige Schlachtkörperverfettung, welche neben der genetischen Veranlagung bei den Landschafrassen (NÜRNBERG et al. 2001, SANTOS-SILVA et al. 2002, DE VRIES et al. 2003, SEIBERT et al. 2004, PARAPONIAK und KAWECKA 2004, POMPAROBORZYŃSKI und KĘDZIOR 2006) auch auf die Gestaltung der Lämmerfütterung in der vorliegenden Untersuchung (hoher Heuanteil bei gleichzeitig geringer Konzentratfuttergabe) zurückzuführen ist. Die Schlachtkörper hatten einen um ca. 3 Prozentpunkte geringeren Anteil wertvolle Teilstücke als die der Lämmer der Kamieniecka-Rasse (SZCZEPAŃSKI et al. 2002a). Beachtenswert ist die deutlich günstigere Gewebezusammensetzung der Keule. Im Vergleich zu Mastlämmern der Rassen Merino und Kamieniecka weist dieses Teilstück zwar einen um 3 bis 5 Prozentpunkte höheren Knochenanteil auf. Der Fettanteil ist jedoch um das 2- bis 3 fache niedriger bei gleichzeitig höherem Fleischanteil (1,5 bis 6 Prozentpunkte). Folglich wirken sich die ermittelten Gewebeanteile bei den Skudden-Lämmern positiv auf das Fleisch-FettVerhältnis (14:1) gegenüber den deutlich engeren Relationen bei Merinolämmern (6,6:1) und den Lämmern der Kamieniecka-Rasse (4,3:1) aus. Über ein vergleichbar günstiges Fleisch : Fett Verhältnis berichten PARAPONIAK und KAWECKA (2004) von in Polen untersuchten Lämmern von Alpenschafrassen.

Die Fleischqualität der Lämmer der Schafrasse Skudden ist hoch zu bewerten. Diese Einschätzung stützt sich auf einen hohen Grad der physiologischen Reife, bewertet nach dem Wasser-Eiweiß-Verhältnis (PARAPONIAK und KAWECKA 2004, BRZOSTOWSKI und TAŃSKI 2006), sowie auf den $\mathrm{pH}_{24}$-Wert, der in dem für optimal gehaltenen Bereich von 5,5-5,9 liegt (SCHIEFFER und SCHARNER 1977). Ein wesentliches von den Verbrauchern geschätztes Fleischmerkmal bei den Skudden-Lämmern ist der sehr niedrige Gehalt an intramuskulärem Fett. Diese Fettfraktion wird während der Zubereitung des Fleisches (z. B. Braten) nicht vermindert. Dadurch hat das Skudden-Lammfleisch einen gesundheitlichen Vorteil (MILEWSKI 2006). Beim intramuskulärem Fettgehalt der Skudden das Lammfleisch anderer Rassen (SZCZEPAŃSKI et al. 2003 BRZOSTOWSKI und TAŃSKI 2006) und ähnelte dem Fleisch der Lämmer im Alter von 50 Tagen (KŁOBUKOWSKI et al. 2002, BRZOSTOWSKI et al. 2004, BRZOSTOWSKI et al. 2006a).

Auch der Energiegehalt des Fleisches ist retativ niedrig und er liegt zwischen den Werten von Lämmern anderer Rassen im Alter von 50 (BRZOSTOWSKI und TAŃSKI 2006) und 100 Lebenstagen (BRZOSTOWSKI et al. 1999). Die dunkle Fleischfarbe lässt sich teilweise mit 
dem geringen Anteil an intramuskulärem Fett erklären (HEYLEN et al. 1998), wird aber wahrscheinlich auch durch den relativ niedrigen Vitamin E-Gehalt mit verursacht. Die gewünschte helle Farbe ist eher durch eine höhere Konzentration dieses Vitamins, welches ein wichtiges Antioxidans im Muskelgewebe ist, bedingt (WOOD et al. 2003). Bei Rindfleisch wird, um einen optimalen Antioxidationsstatus zu erreichen, ein Vitamin E-Gehalt in der Größenordnung von 3-3,5 $\mu \mathrm{g} / \mathrm{g}$ für erforderlich gehalten (ARNOLD et al. 1993). Bei Lammfleisch liegt die Vitamin E-Konzentration oft unter diesem Niveau, insbesondere dann, wenn die Fütterung mit Kraftfutter erfolgt (WOOD et al. 2003).

Das untersuchte Skudden-Lammfleisch enthält einen 1- bis 1,5-fach geringeren Cholesteringehalt gegenüber dem Lammfleisch anderer Rassen, die im Alter von 50 Tagen (BRZOSTOWSKI et al. 2004, BRZOSTOWSKI et al. 2006a) bzw. 100 Tagen (BRZOSTOWSKI und TAŃSKI 2006) geschlachtet wurden. Das Skudden-Fleisch ist somit sehr arm an Cholesterin. Dieser Befund kann teilweise auf die frühere Geschlechtsreife der Skudden zurückzuführen sein. Eine intensive Produktion von Geschlechtshormonen ist mit wesentlichen Änderungen im Cholesterinstoffwechsel verbunden, weil Cholesterin als ihr Präkursor fungiert (MURRAY et al. 2006), was folglich dazu führen kann, dass das Cholesterin periodisch in kleinerem Ausmaß im Gewebe abgelagert wird. Andererseits ist aber auch von großer Bedeutung, dass die Fütterung der Muttertiere und Mastlämmer ausschließlich mit Grünfutter, Heu und Hafer erfolgte, was sich sowohl indirekt über die Muttermilch wie auch direkt auf die Senkung des Cholesterinspiegels im Lammfleisch auswirken könnte (SANTOS-SILVA et al. 2002). Das Ergebnis solch einer Fütterung ist eine geringere Verfettung der Schlachtkörper, was im Endeffekt den Cholesteringehalt im Lammfleisch beeinflusst, denn diese Merkmale weisen eine positive Korrelation auf dem Niveau von $r=0,65$ auf (HONIKEL 2000).

Ein besonderes Merkmal von Skudden-Lammfleisch ist der kleine Profildurchmesser der Muskelfasern. Der Durchmesser ist ca. 27 bzw. 12 Prozentpunkte geringer als der bei den Muskelfasern von Lämmern der Pommerschen Rasse sowohl im Alter von 100 (BRZOSTOWSKI und TAŃSKI 2006) als auch 50 Tagen (BRZOSTOWSKI et al. 2006). Dies ist ein wertvolles Ergebnis, denn der geringere Muskelfaserdurchmesser kann sich positiv auf die Bekömmlichkeit und die Verdauung dieses Fleisches (KŁOBUKOWSKI et al. 2002) auswirken.

Das Aminosäuremuster des Fleischproteins von Skudden-Lämmer unterscheidet sich kaum von dem der Lämmer der Pommerschen Rasse (BRZOSTOWSKI und TAŃSKI 2006). Jedoch das Fettsäureprofil des intramuskulären Fett ist in Bezug auf das Verhältnis von DFASäuren (Säuren, die den Cholesterinspiegel im Blut reduzieren) zu OFA-Säuren (Säuren, die den Cholesterinspiegel im Blut erhöhen) günstiger.

Im Vergleich zum Fleisch der Lämmer der Kamieniecka Rasse, die im gleichen Alter geschlachtet wurden (SZCZEPAŃSKI et al. 2003), enthält das Fleisch von Skudden-Lämmern mehr mehrfach ungesättigte Säuren, darunter Linolsäure $C_{18: 2^{\prime}}$ die als ungesättigte Fettsäure aus der Gruppe der n-6-Fettsäuren bei der Synthese u.a. von Eicosanoiden und Gewebehormonen, die wesentliche Funktionen im Körper erfüllen, unentbehrlich ist (MILEWSKI 2006). Neben dem Fütterungseinfluss auf den Gehalt an PUFA im Lammfleisch (NÜRNBERG et al. 2001, GRUSZECKI et al. 2004) zeichnen sich auch andere Landschafrassen durch einen höheren Gehalt an PUFA aus (BRZOSTOWSKI und TAŃSKI 2006, BRZOSTOWSKI et al. 2006b, POMPA-ROBORZYŃSKI und KĘDZIOR 2006). Dies bestätigt die hohe Gesundheitsqualität des Skudden-Lammfleisches, was auch mit den Untersuchungs- 
ergebnissen von NUERNBERG et al. (2008) übereinstimmt. Vor diesem Hintergrund ist auch der hohe Vitamin A-Gehalt heraus zu stellen. Dieser ist 3-fach höher im Vergleich zum Schweinefleisch (FLIS et al. 2007), welches nach wie vor die Hauptposition bei den konsumierten Fleischarten einnimmt.

Von der guten Fleischqualität von Skudden-Lämmern zeugen des Weiteren die hohen Noten für die sensorischen Merkmale, die im Vergleich zu den Lammfleischbewertungen anderer Rassen in einem höheren Bereich liegen (PARAPONIAK und ROBORZYŃSKI 2001, PARAPONIAK und KAWECKA 2004, BRZOSTOWSKI und TAŃSKI 2006, BRZOSTOWSKI et al. 2006b, POMPA-ROBORZYŃSKI und KĘDZIOR 2006). Anhand der an 100 Tage alten Lämmern durchgeführten Untersuchungen kann man feststellen, dass der Schlachtwert der SkuddenLämmer ein gutes Niveau aufweist. Die relativ niedrige Lebendmasse der Lämmer im Vergleich zu den Lämmern von Fleisch- und Wollfleischrassen im vergleichbarem Alter wird in bestimmten Umfang durch eine geringe Schlachtkörperverfettung und eine wesentlich günstigere Gewebezusammensetzung der Keulen kompensiert. Die Fleischqualität von Skudden-Lämmern übertrifft die anderer Schafrassen. Das Fleisch zeichnet sich durch eine dunkle Farbe und spezifische sensorische Merkmale aus, dank denen es mit Wildfleisch vergleichbar ist. Der geringe Fett- und Cholesteringehalt, die hohe Vitamin A-Konzentration und das günstige Fettsäureprofil vom intramuskulären Fett sind ernährungsphysiologische und gesundheitliche Vorteile des Skudden-Lammfleisches. Die feinfaserige Muskelstruktur weist darauf hin, dass dieses Fleisch leicht verdaulich und gut bekömmlich ist.

Die erzielten Ergebnisse befürworten eine breitere Nutzung der Skudden für die Lammfleischerzeugung, zumal sich diese Rasse durch eine hohe Fruchtbarkeit auszeichnet, die sich sowohl im Ablammergebnis als auch in einer erhöhten Geburtenhäufigkeit (Ablammungen pro Jahr) widerspiegelt (FISCHER et al. 1994). Ferner kann man Skudden aufgrund ihrer niedrigen Anforderungen an Haltungsbedingungen und Fütterung als ein sich »selbst bedienendes Schaf« bezeichnen. Diese Argumente sprechen deutlich für eine Restitution dieser anspruchslosen Schafrasse in Ermland und Masuren.

\section{Literatur}

Arnold RN, Arp SC, Scheller KK, Williams SN, Schaefer DM (1993) Tissue equilibration and subcellular distribution of vitamin E relative to myoglobin and lipid oxidation in displayed beef. J Anim Sci 71 1, 105-18

Baryłko-Pikielna N (1975) The dryft of the ford sensory analysis. PWN Warszawa [in Polish]

Brzostowski H, Niżnikowski R, Milewski S (2006a) Composition and properties of meat of Pomerania, purebred lambs and their crossbreeds with Berrichon du Cher and Charolaise. Arch Tierz 49, 494-501

Brzostowski H, Sowińska J, Tański Z (2006b) Slaughter value and quality of meat from Pomeranian lambs and crossbreds by Blackface and Charolaise rams. Anim Sci Papers Rep 24 Suppl 23, 53-60

Brzostowski H, Tański Z (2006) Nutritional value of the meat of Pomeranian breed lambs and crossbreeds of Blackheaded and Texel rams. Arch Tierz 49 Special Issue, 345-52

Brzostowski H, Tański Z, Sowińska J (1999) Some parameters of slaughter value and meat quality of Kamieniec rams and their crossbreeds. Biul Nauk (Acta Acad Agricult Tech Olstnensis) 5, 193-202 [in Polish]

Brzostowski H, Tywończuk J. Tański Z (2004) Indexes of nutritive value of meat obtained from Pomeranian lambs and crossbreeds of Pomeranian ewes with meat breed rams. Arch Tierz 47 Special Issue, 175-82

De Vries F, Hamann H, Distl O (2003) Estimation of genetic parameters in lamb sheep breeds. Arch Tierz 47, 351-8 [in German] 
Duchev Z, Distl O, Groeneveld E (2006) Early warning system for loss of diversity in European livestock breeds. Arch Tierz 49, 521-31

Fischer A, Leipnitz W, Schalitz G (1994) Investigations for selected parameters of efficiency and characterization of exterieur of Skudde. Arch Tierz 37, 651-8 [in German]

Flis M, Sobotka W, Antoszkiewicz Z, Lipiński K, Zduńczyk Z (2007) Effect of husked and naked oat used in the diets supplemented with linseed oil on the growth performance of pig, carcass and meat quality. Arch Tierz 50 Special Issue, 161-71

Grau R, Hamm R, Baumann A (1953) About the water-binding capacity of the dead mammalian muscle. I. Communication: The influence of pH on water-binding of comminuted beef muscle. Biochem Z 325 (1), 1-11 [in German]

Gruszecki T, Junkuszew A, Lipecka C, Kamińska A, Szymanowska A, Patkowski K (2004) Fatty acid composition in sheep milk and muscle tissue of lamb fed with protective fat-supplemented fodder. Arch Tierz 47 Special Issue, 183-8

Heylen K, Suess R, Freudenreich P, Lengerken von G (1998) Relationship between intramuscular fat content and meat quality in lambs with emphasis on sensory characteristics. Arch Tierz 41, 111-22 [in German]

Honikel K (2000) Relationship between contents of cholesterol and fat in meat cuts. Proceedings 46th ICoMST Argentina 2, 620-1

Kłobukowski J, Brzostowski H, Tański Z, Wiśniewska-Pantak D, Sowińska J (2002) The quality and nutritive value of the meat protein of various lambs genotypes. Pol J Food Nutr Sci 11, 441-5

Knabe P, Fischer A, Leucht W (1988) Skudde - a breed study. Arch Tierz 31, 84-90 [in German]

Krische G (1992) 50 Jahre Haltung von Skudden im Leipziger Zoo. Panthera, Leipzig, 27-30

Kurt G, Brandt HR (2008) Development and characterization of lamb's coats of East Prussian Skudden and Rough-coated Pomeranian Landrace sheep. Arch Tierz 51, 467-78

Milewski S (2006) Health-promoting properties of sheep products. Med Weter 5, 516-9 [in Polish]

Murray R, Granner D, Mayes P, Rodwell V (2006) Harper's Illustrated Biochemistry. MCGRAW HILL

Nawara W, Osikowski M, Kluz I, Modelska M (1963) Cost evaluation of rams based on progeny testing in ram testing stations Institute of Animal Production. PWRiL Warszawa 166, 48-58 [in Polish]

Nürnberg K, Grumbach S, Nürnberg G, Hartung M, Zupp W, Ender K (2001) Influence of breed and production system on meat quality and fatty acid composition in lamb muscle. Arch Tierz 44 Special Issue, 351-60

Nürnberg K, Grumbach S, Zupp W, Hartung M, Nürnberg G, Ender K (2001) Enhancing of n-3 fatty acids and conjugated fatty acid in lamb meat by keeping on pasture [in German] 9, 120-2

Nürnberg K, Fischer A, Nürnberg G, Ender K, Dannenberger D (2008) Meat quality and fatty composition of lipids in muscle and fatty tissue of Skudde lambs fed grass versus concentrate. Small Rumin Res 74 (1-3), 279-83

Paraponiak P, Kawecka, A (2004) Raising Alpinie breed of sheep for meat and milk under the environmental conditions of the Beskid Sadecki Mountains. Arch Tierz 47 Special Issue,198-206

Paraponiak P, Roborzyński M (2001) The use of Tiroler Bergschaf und Weisses Alpenschaf for the production of slaughter lambs in Polish mountainous areas. Arch Tierz 44 Special Issue, 391-7

Peisker KV (1964) A rapid semi-micro method for preparation of methyl ester from triglycerides using chloroform, methanol, sulphuric acid. J Am Oil Chem Sco 41, 87-8

Pompa-Roborzyński M, Kędzior W (2006) Effects of crossbreeding and different feeding systems on slaughter value and meat quality of lambs reared in natura pastures of the Beskid Sadecki Mountains. Arch Tierz 49 Special Issue, 268-74

Rettenmaier R, Schüep W (1992) Determination of vitamins A and E in liver tissue. Int J Vitam Nutr Res 62, 312-7

Rhee KS, Dutson TR, Smith GC, Hostetler RL, Reiser R (1982) Cholesterol content of raw and cooked beef longissimus muscles with different degrees of marbling. J Food Sci 47, 716-9

Sambraus HH (1996) Atlas of livestock breeds, 5th ed, Ulmer, Stuttgart, Germany [in German] 
Santos-Silva J, Bessa RJB, Santos-Silva J (2002) The effect of genotype, feeding system and slaughter weight on the quality of light lambs. Livest Prod Sci 77, 187-94

Schieffer G, Scharner E (1977) Influence of age, liveweight, sex and breed on selected quality parameters of lamb meat [in German]

Seibert B, Gauly M, Erhardt G (2004) Productivity of differentsheep breeds in extensive pasture management. Arch Tierz 47 Special Issue, 142-52

Szczepański W, Czarniawska-Zając S, Milewski S (2002a) Carcass quality of lambs of Kamieniecka ewes and crossbreds from Charolaise rams. Zesz Nauk Prz Hod 63, 101-5 [in Polish]

Szczepański W, Milewski S, Czarniawska-Zając S (2002b) Slaughter value of lambs of Kamieniecka ewes and ceossbreds from Charolaise rams. Zesz Nauk Prz Hod 63, 107-11 [in Polish]

Szczepański W, Milewski S, Czarniawska-Zając S (2003) Quality of meat from lambs out of Kamieniecka ewes and crossbreeds by Charolaise rams. Ann UMCS Lublin XXI 1 18, 135-40

Śliwa Z (1957) Breeding of sheep. PWN Łódź-Poznań [in Polish]

Wood JD, Richardson RI, Nute GR, Fisher AV, Campo MM, Kapasidou E, Sheard PR, Enser M (2003) Effect of fatty acid on meat quality: a review. Meat Sci 66, 21-32

Żegarska Z, Markiewicz K, Smoczyński S (1979) Composition of fatty acid in muscle fat and depot fat of rabbits. Zesz Nauk ART Olsztyn 15, 167-77 [in Polish]

Received 30 January 2009, accepted 18 January 2010.

Corresponding author:

STANISŁAW MILEWSKI

email: stanislaw.milewski@uwm.edu.pl

Department of Sheep and Goat Breeding, University of Warmia and Mazury in Olsztyn, ul. Oczapowskiego 5, 10-719 Olsztyn, Poland 